\title{
Using eye tracking to differentiate student difficulties reasoning with data
}

\author{
Raymond Zich and Rebecca Rosenblatt \\ Department of Physics, Illinois State University, Normal, IL, 61790
}

\begin{abstract}
Findings from a past project studying students in algebra-based physics courses indicate significant issues working with graphed and pictured data. Students overuse reasoning schemes that work for one cause - one effect data but do not work for multiple causes. Students make incorrect claims like, "If variable A is unchanged and variable B does change than variable A must not affect variable B." In this study, we use eye tracking data to investigate students' attention to the variables in the graphs and pictures. We observe different student gaze patterns on questions answered incorrectly vs. correctly for single-trend questions. This demonstrates that control-of-variables (and not just logical reasoning) affects student skills interpreting this data. In addition, we present other results. For example, the pictorial data requires significantly less attention time for students to analyze than does the single-trend graphed data even though these present the same data sets.
\end{abstract}

\section{INTRODUCTION}

Past research including our own [1-6], shows that students have difficulties with logical reasoning involving dependence among multiple variables. In addition they have difficulties applying control of variables [1-6]. Data indicate approximately $70 \%$ of students enrolled in the algebra based physics course at Illinois State University incorrectly answer questions about uncontrolled data in both graph and picture form. (See Figure 1, graph B and picture D for examples of uncontrolled data, i.e. data where more than one variable is changed at once.) Instead of the correct multivariable reasoning, students use reasoning that accounts only for single cause - single effect situations, without regard to additional variables. For example, (see Fig. 1 - graph B) students reason that because mass and period both change mass affects the period, which does not account for changes to pendulum length shown in the legend.

Furthermore, students struggle with questions about a variable which was unchanged in graph and/or pictorial data. When a variable is controlled (i.e. unchanged to observe the effect on another variable) and students are asked about that variables effect they respond incorrectly. $70 \%$ of students claim if a variable is unchanged and another variable changes, the first variable does not affect the second variable. An example of this is shown in Figure 1 graph A and picture $\mathrm{C}$, where students claim the data shown is evidence that mass does not affect the period. This reasoning is not isolated to mass and period of a pendulum, but is a general pattern of incorrect assertions.

In addition to the errors seen with "single-trend" data sets, students display these same errors when working with larger "multi-trend" data sets. For example in Figure 1 graph E, the multi-trend data indicates that pods affect widgets, however, $70 \%$ of students report that comparisons between certain data pairs (like those indicated by $\mathrm{B}$ and $\mathrm{C}$ or those indicated by $\mathrm{A}$ and C) demonstrate that pods do not affect widgets. While the data displayed is controlled, the comparisons students make among the data are often not controlled and this leads to the same incorrect answers and reasoning described above.

These results are summarized by Rosenblatt and Perrone:

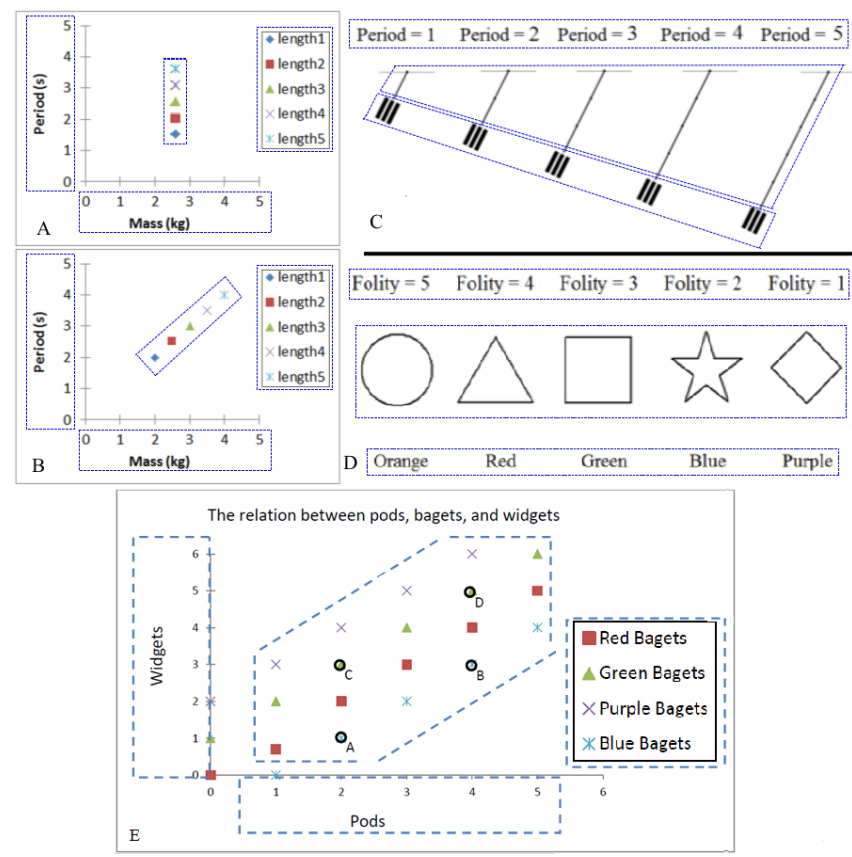

FIG. 1. Examples of data presented to students. A. Controlled single-trend graph; B. Uncontrolled graph; C. Pictorial pendulum data; D. Pictorial shapes data; E. Multi-trend graph. Areas of interest (AOIs) for eye tracker analysis are shown by the dotted lines which enclose certain regions on the images but were not present in the images shown to students.

"Students make persistent and consistent logical mistakes concluding 'since B changes and A does not, then A must not affect/change B' or 'since A changed and B changed, A must affect B.' ...These findings suggest students' errors are rooted in their understanding of control of variables and ability to reason in a logically correct fashion.'[2]

While these studies clearly illustrate the student difficulties working with multivariable data, these studies cannot distinguish between student errors due to control of variables and errors due to logical reasoning. The inability to distinguish between the two types of error originates from the fact that visibility of a variable does not guarantee students are attend- 
ing to it. While it is clear that many students have difficulty with this data, and that one of these two issues is the cause, the best course of instructional intervention is unclear because it is unknown which of the issues causes the difficulty. In this paper, eye tracking is used to investigate the actual visual attention of students while working on these graph and pictorial questions. This visual attention is then used to distinguish between students who are not properly attending to the multiple variables, and therefore are not controlling the variables, and students who are properly attending to the multiple variables but are still responding incorrectly and therefore are having reasoning issues. In this study we define "not properly attending" as distributing gaze time across the variables differently than the averages needed to answer the question correctly.

\section{METHODOLOGY: VISUAL ATTENTION AND EYE TRACKING THEORY \& EXPERIMENT DESIGN}

Studies have shown that eye movements can reflect cognitive processes when students are answering questions [9]. In this study the strong eye-mind theory of Just and Carpenter is presumed [10]. This theory states that cognitive processing of an object being viewed occurs simultaneously with the viewing of the object. In our study this theory was interpreted to mean that time spent by a student looking at an area on a graph or picture reflected the cognitive attention of the student to that information.

A Tobii X2-60 eye tracker was used to record the position of students' gazes on the screen while they were looking at slides of graphed and pictorially presented data such as shown in Figure 1. This eye tracker uses paired infrared cameras to record the position and motion of the pupil, then calculates the location of the gaze point on the screen from a set of calibration points it takes at the beginning of each interview. (It displays a circle in each corner and the middle of the screen for a second or two and records each student's pupil placement for those known screen spots.) The students use a chin and forehead rest to ensure that their head remains in the same position throughout the interview and therefore that the calibration data is valid for the whole set of images.

The software OGAMA was used to link the data from the Tobii to the displayed image at that time. After the data is collected OGAMA will display the gaze pattern and saccades (eye movements) as a real-time video on the images shown and statistical data can be collected to calculate average amounts of time that students fixed their gaze in certain regions of the images. These regions are called "areas of interest" or AOIs. Figure 1 shows examples of the different AOIs used for the different types of data that students saw. The AOIs were selected based on the research question and focus on the variables used to display the data.

Students were recorded as they considered a set of data in graph or pictorial form and selected an answer for a multiple choice question about a variable's affect on another variable in the data set. (The question was displayed to the left of the graph or picture.) When they had an answer in mind, the students pressed a button to advance the slide and then explained their reasoning and choice to the interviewer. The average amount of time spent looking at each variable (AOI) for correct responses vs. incorrect responses indicates whether control of variables issues or logical reasoning issues predominate. If the students AOI gaze pattern is the same whether they answer the question correctly or incorrect, then logical reasoning issues are the attributed difficulty. If, however, the students AOI gaze patterns are different when they answer the question correctly vs. incorrectly then attention to the variables is different and control of the variables is the difficulty.

18 different questions on the single-trend graphs and pictorially represented data were presented to students in two sets so that students could sit back from the eye tracker and take a break between sets. A third set of seven multi-trend questions addressed these types of graphs.

There were four types of images used to display relationships between variables. Single-trend graphs (STG) displayed data on pendulum length, mass, and period. Pictorial pendulums displayed data on pendulum length, mass, and period. Pictorial shapes displayed data on shapes, colors, and 'folity'. Multi-trend graphs (MTG) displayed data on bagets, pods, and widgets. Bagets, pods, widgets, and folity were words chosen because they lack physics context. This was done so the results would not be affected by prior physics knowledge of the students. The single-trend and multi-trend graphs were divided into the AOIs Xaxis, Yaxis, Datapoints, and Legend. The pendulum slides were divided into the AOIs Length, Mass, and Period, and the shapes slides were divided into the AOIs Shapes, Colors, and Folity. (Figure 1 illustrates the four type of images and the AOIs for each.) On each slide, students were asked a multiple choice question about a variable's effect on period, folity, or widgets (whichever was relevant for that question type). For example, pendulum pictures and graphs had this wording for a length question: Which of the following best describes the relationship between length and period? a) Period depends on length (the length changes the period); b) Period does not depend on length (the length does not change the period); c) There is not enough information given to determine if there is, or is not, dependence.

Data was collected from 18 students selected from a traditionally taught algebra-based physics course, post instruction, between the 9 th and 12 th weeks of the semester. Randomly selected students had the option of doing this interview or a different assignment. Over $90 \%$ of students asked agreed to do the interview.

\section{GAZE PATTERN ANALYSIS}

Considering the average fixation time for the four different data set types (See Table I for this data) several things of interest become clear. First, the average gaze times for students looking at the pictorially presented data was significantly less than time spend for the single-trend graphs which 
TABLE I. Average Total Gaze Time

\begin{tabular}{ccccc}
\hline Answers & STG $(\mathrm{ms})$ & MTG $(\mathrm{ms})$ & Pendulum $(\mathrm{ms})$ & Shapes $(\mathrm{ms})$ \\
\hline Correct & 7136 & 8983 & 5304 & 4702 \\
Incorrect & 6706 & 9226 & 5445 & 5470 \\
Total & 6921 & 9105 & 5374 & 5086
\end{tabular}

was in turn significantly less than time spent on the multitrend graphs. The average time for pictured data was 5230 ms vs. $6921 \mathrm{~ms}$ for single-trend graphs and $9105 \mathrm{~ms}$ for multi-trend graphs $(F=4.879, p=0.028$ and $F=4.596$, $p=0.033$ ). For comparison, Just and Carpenter found that gaze times for single comparison tasks are about $200 \mathrm{~ms}$ [11]. Multi-trend graphs displayed more data and thus a longer gaze time would be expected, but the single-trend graph has the same set of information as the pictured data. It is possible that this additional gaze time for graphs represents higher level of difficulty even though the average percentage of correct responses is similar. Second, for multi-trend graphs and pictorially presented data, students who answered incorrectly looked at the images for more time (384 ms on average) than students who answered correctly. However, for single-trend graphs students who answered correctly spent an average of $430 \mathrm{~ms}$ more time looking at the graph. This suggests that the different data presentations require different mental tasks.

When each area of interest in the different data presentations is considered, additional findings are apparent (see Tables II-V). Each table displays the average fixation time and the standard deviation in each AOI for that type of data representation. Also, each students' total gaze time was calculated and the percentages of their gaze time in each AOI was found to show a pattern of time spent observing each AOI. The tables present this information broken up by whether the student was correct or incorrect on the questions.

For the single-trend graphs, students unevenly divided their gaze time among AOIs; the range of time spent viewing the AOIs was from $1116 \mathrm{~ms}$ (19\%) to $2298 \mathrm{~ms}$ (32\%). Students who answered correctly spent the largest percentage of their time looking at the legend. Correct students viewed the legend $32 \%$ of the time vs. only $20 \%$ of the time for incorrect students. (Incorrect students divided this 'extra time' between the data points and y axis.) Over all, the gaze pattern for stu-

TABLE II. Gaze Time per AOI for Single-Trend Graphs. Percentages are calculated by taking the fraction of gaze time spent in each AOI compared to the total gaze time for each student.

\begin{tabular}{ccccc}
\hline Answers & Xaxis (ms) & Yaxis (ms) & Datapoints (ms) & Legend (ms) \\
\hline Correct & 1336 & 1542 & 1960 & 2298 \\
stdev & 1190 & 1082 & 1420 & 2507 \\
\% of Total & $19.3 \%$ & $25.3 \%$ & $23.5 \%$ & $31.8 \%$ \\
\hline Incorrect & 1116 & 1965 & 2227 & 1398 \\
stdev & 787.4 & 1869 & 2944 & 1565 \\
\% of Total & $18.6 \%$ & $30.5 \%$ & $30.4 \%$ & $20.4 \%$
\end{tabular}

TABLE III. Gaze Time per AOI for Pictorial Pendulum Data

\begin{tabular}{cccc}
\hline Answers & Length (ms) & Mass (ms) & Period (ms) \\
\hline Correct & 1439 & 1665 & 2200 \\
stdev & 1388 & 1761 & 3963 \\
\% of Total & $31.0 \%$ & $32.2 \%$ & $36.8 \%$ \\
\hline Incorrect & 1591 & 1922 & 1931 \\
stdev & 1927 & 1684 & 2835 \\
\% of Total & $32.4 \%$ & $36.2 \%$ & $31.3 \%$
\end{tabular}

dents looking at the single-trend graphs and answering the questions correctly is not significantly different than the gaze pattern for students who answered incorrectly as shown by a chi-squared test $\left(\chi^{2}=4.148, p=0.25\right)$. However, a post hoc t-test does show that attention to the legend variable is different $(F=7.495, p=0.01)$. This shows that control of variables contributes to student difficulties with single-trend graphs. In order for students to answer these questions correctly, they must attend to and control for the legend variable. These gaze patterns show students answer the question correctly when they are attending more to the legend variable, but if they insufficiently attend to the legend, thus control for the legend variable, they will be incorrect.

For the pictorially depicted pendulum data (see Table III), approximately $\frac{1}{3}$ of the viewing time was spent on each of the three AOIs, irrespective of whether the answer given was correct or incorrect. For the pictorially depicted shapes data (see Table IV), the amount of time spent viewing the Folity AOI was about $48 \%$, greater than the time spent viewing either the Shapes or the Colors AOIs which were on average viewed $27 \%$ of the time each. (We believe this extra time spent viewing Folity was caused by students attempting to figure out what the word was.) A chi-squared test for independence of the gaze pattern percentages for correct and incorrect students shows that these gaze patterns on both types of pictured data are the same $\left(\chi^{2}=0.776, p=0.68\right.$ for the pendulum data and $\chi^{2}=0.288, p=0.87$ for the shapes data). Observations of the data do not suggest any large differences for post hoc analysis. This suggests that control of variables is not the cause for student difficulties with these questions, i.e. students attend to the variables in the same fashion when they are incorrect or when they are correct. Therefore reasoning issues - and not attention/inattention to variables - are the cause for

TABLE IV. Gaze Time per AOI for Pictorial Shape Data

\begin{tabular}{cccc}
\hline Answers & Shapes (ms) & Colors (ms) & Folity (ms) \\
\hline Correct & 1310 & 1205 & 2187 \\
stdev & 1120 & 1006 & 1985 \\
\% of Total & $29.8 \%$ & $24.8 \%$ & $45.4 \%$ \\
\hline Incorrect & 1563 & 1348 & 2559 \\
stdev & 2549 & 1787 & 2614 \\
\% of Total & $29.7 \%$ & $22.2 \%$ & $48.1 \%$
\end{tabular}


TABLE V. Gaze Time per AOI - multi-trend Graphs

\begin{tabular}{ccccc}
\hline Answers & Xaxis (ms) & Yaxis (ms) & Datapoints (ms) & Legend (ms) \\
\hline Correct & 1567 & 1390 & 4843 & 1211 \\
stdev & 1190 & 1266 & 3489 & 1506 \\
\% of Total & $18.1 \%$ & $13.0 \%$ & $61.8 \%$ & $7 \%$ \\
\hline Incorrect & 1544 & 1597 & 4797 & 1288 \\
stdev & 1667 & 1296 & 5574 & 1338 \\
\% of Total & $18.7 \%$ & $15.2 \%$ & $59.7 \%$ & $7.4 \%$
\end{tabular}

student difficulties with these items.

For the multi-trend graphs (see Table V), in the case of both correct and incorrect answers students spent the most time viewing the Datapoints AOI, 62\% when correct, and $60 \%$ when incorrect. The time spent viewing the $\mathrm{X}$ axis and $\mathrm{Y}$ axis were roughly the same, around $18 \%$ for the $\mathrm{X}$ axis and $14 \%$ for the $\mathrm{Y}$ axis, and very little time, only $7 \%$, was spent looking at the Legend for both correct and incorrect students. A chi-squared test for independence of the gaze pattern percentages for correct and incorrect students shows that these gaze patterns are the same $\left(\chi^{2}=0.244, p=0.97\right)$.

For the multi-trend graphs students clearly concentrate on the data. Unlike with single-trend graphs significant attention to the legend variable seems to be unnecessary for correct interpretation and the $\mathrm{x}$ and $\mathrm{y}$ axis also need less attention. The researchers believe that this gaze data actually shows that for multi-trend graphs students do not need to attend to the definitions of the variables for a large amount of time. Attention to the variables can be accomplished through observation of the data on a multi-trend graph. For this reason, these gaze data do not necessarily inform on which issue students are having. It is possible that reasoning is the main issue. However, if attention patterns within the Datapoints AOI are different control of variables may be the issue.

\section{CONCLUSION}

The goal of this project was to use eye tracking data to identify whether logical reasoning difficulties or control of variables issues caused student errors in answering questions about dependence of variables in visually presented data sets. The eye tracking data collected shows that students who correctly answered questions related to single-trend graphs spent significantly longer attending to the legend variable than students who were incorrect; this is an indication that control of variables and inattention to the legend is the cause of errors for single-trend graphs. Pictured data for both pendulums and shapes does not show this same variation. Gaze patterns across AOIs for pictorial data are the same whether or not students answer the question correctly; this is an indication that logical reasoning issues, and not attention to variables/control of variables, are the cause for errors with pictorial data.

It is important to note that the gaze pattern differences or similarities are what was observed. Connecting this to control of variables and logical reasoning assumes that correct responses require both correct control of variables and correct logical reasoning, and that inattention to variables means incorrect control of variables.

Over all, this eye tracking data reports that for pictorial data students do not have the same control of variables issues that they show with single-trend graph data. In addition, the eye tracking data shows that while pictorial and single-trend data sets have the same data - and thus it might be assumed that they require the same cognitive processes to work with that data - this is not the case. The pictorial data requires significantly less attention time for students to analyze than does the single-trend graph data.

Lastly, eye tracking data with multiple variables show that students have the same gaze patterns on AOIs for correct and incorrect responses. This may indicate that students have logical reasoning issues with these graphs like they do with the pictorial data. However, because of the lack of time spent attending to the variables over the data points, the researchers are hesitant to make this claim; it may be that the data points themselves allow for attention to the different variables and future research into saccade movements is planned to investigate this. It is clear that even though the multi-trend graphs are slightly easier, i.e. students are slightly more likely to answer correctly, the multi-trend graphs take significantly more time than the single-trend graphs for students to analyze.

\section{ACKNOWLEDGMENTS}

Special thanks to the PERTG for a 2016 Scholar-inResidence Grant and a 2017 Travel Award. Thanks are due to Sanjay Rebello for his guidance and encouragement on the experimental design and to Rebecca Lindell for her advice.
[1] R. J. Rosenblatt, PERC Proceedings, (2014).

[2] R. J. Rosenblatt and J. Perrone PERC Proceedings, (2016).

[3] A. M. Bogdan and A. F. Heckler, PERC Proceedings, (2013).

[4] A. E. Lawson, Journal of Research in Teaching Science 15 (1), 11-24 (1978).

[5] A. Bodreaux, P. S. Shaffer, P. R. L. Heron, and L. C. McDermott, Am. J. Phys. 76, 163-170 (2007).
[6] L. C. McDermott and the Physics Ed. Group at the Univ. of Washington, Physics by Inquiry Wiley, New York, (1996).

[7] A. J. Ross, Review of Ed. Research 58, $405-437$ (1988).

[8] R. J. Beichner, Am. J. Phys. 62, 750-762 (1994).

[9] D. E. Meltzer, Am. J. Phys. 73 (5), 463-478 (2005).

[10] X. Wu, PERC Proceedings, (2015).

[11] M. A. Just and P. A. Carpenter, Cognitive Psychology, 8 (4), 441-480 (1976). 